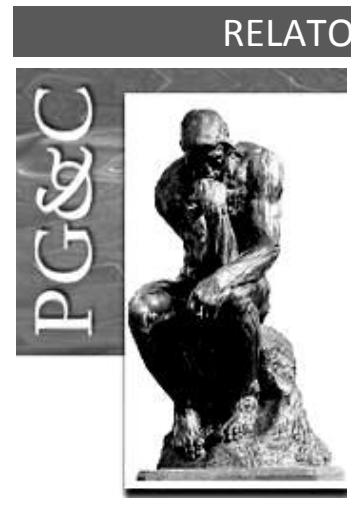

\title{
AVALIAÇÃO DA MATURIDADE DA GESTÃO DO CONHECIMENTO NA ADMINISTRAÇÃO PÚBLICA
}

\author{
Vanessa dos Santos \\ Mestre em Contabilidade pela Universidade Federal de \\ Santa Catarina, Brasil. \\ E-mail: assemav.san@gmail.com \\ Rogério Cid Bastos \\ Doutor em Engenharia da Produção pela Universidade Federal de Santa \\ Catarina, Brasil. Professor da Universidade Federal de Santa Catarina, \\ Brasil. \\ E-mail: rogerio@inf.ufsc.br
}

\begin{abstract}
Resumo
Este estudo tem como objetivo avaliar a maturidade da gestão do conhecimento na Administração Pública. A importância de se medir o nível em que se encontra a gestão do conhecimento em uma organização se dá pelo constante aprimoramento no processo de identificar, criar, armazenar, compartilhar e aplicar o conhecimento organizacional, de modo que a memória organizacional não se perca ao longo do tempo ou mesmo que seja desprezada pela introdução de novos conceitos e procedimentos. Utiliza como fundamento a aplicação da metodologia desenvolvida por Helou (2015), a qual adaptou os modelos propostos pela APO (2009) e MGCAPB (Batista, 2012). O modelo utilizado consiste na aplicação de um questionário, o qual se desenvolve em nove dimensões avaliativas, quais sejam, liderança; pessoas; processos; tecnologia; processo de GC; aprendizagem e inovação; resultados da GC; cultura organizacional; e arcabouço legal. O objeto de estudo é uma Instituição Pública Catarinense, trata-se, portanto, de uma pesquisa de abordagem qualitativa de cunho exploratório descritivo. Os resultados obtidos apontam que a instituição pública avaliada enquadra-se no nível de expansão, com 102 pontos no total, ou seja, ela tem consciência da gestão do conhecimento na organização. Entretanto, ainda é um processo incipiente, uma vez que se observa haver práticas de gestão do conhecimento em algumas áreas de forma isolada, como se pode inferir das pontuações intermediárias obtidas em cada dimensão.
\end{abstract}

Palavras-chave: Gestão do Conhecimento. Administração Pública. Maturidade da Gestão do Conhecimento.

\section{MATURITY ASSESSMENT OF KNOWLEDGE MANAGEMENT IN PUBLIC ADMINISTRATION}

\begin{abstract}
This study aimed to evaluate the maturity of knowledge management in Public Administration. The importance of measuring the level of knowledge management in an organization is due to the constant improvement in the process of identifying, creating, storing, sharing and applying organizational knowledge, so that organizational memory is not lost throughout of the time or even be neglected by the introduction of new concepts and procedures. The application of the methodology developed by Helou (2015), which adapted the models proposed by APO (2009) and MGCAPB (Batista, 2012) was used as the foundation. The model used is the application of a questionnaire, which is developed in nine evaluative dimensions, namely, leadership; people; processes; technology; process; learning and innovation; GC results; organizational culture; and legal framework. The object of study was a Catarinense Public Institution, it is therefore a qualitative research with a descriptive exploratory character. The results show that the public institution evaluated is in the level of expansion, with 102 points in total, that is, it is aware of the knowledge management in the organization. However, it is still an incipient process, since
\end{abstract}

Perspectivas em Gestão \& Conhecimento, João Pessoa, v. 9, n. 1, p. 24-41, jan./abr. 2019. DOI: http://dx.doi.org/10.21714/2236-417X2019v9n1p24

http://periodicos.ufpb.br/ojs2/index.php/pgc. ISSN: 2236-417X. Publicação sob Licença (cc) EY-NC-ND 
there are practices of knowledge management in some areas in isolation, as can be inferred from the intermediate scores obtained in each dimension.

Keywords: Knowledge Management. Public Administration. Knowledge Management Maturity.

\section{INTRODUÇÃO}

A Administração Pública deve situar os cidadãos no centro de suas decisões, acompanhando as transformações da sociedade e suas demandas, de modo a aumentar a satisfação da população e dos grupos de interesses, utilizando-se de boas práticas de gestão e identificando os pontos de melhorias para obter resultados mais satisfatórios. Isso obriga a Administração Pública a conhecer quais ativos estão relacionados com a informação, o conhecimento, o talento e a aprendizagem existentes na organização, para poder criar, transformar e transmitir conhecimento, de modo que os ativos intangíveis possam ser geridos de forma eficiente e eficaz, em outras palavras, conhecer e estruturar a gestão do conhecimento (JUÁREZ; CERVANTES, 2012).

A gestão do conhecimento foi definida por Pardo et al. (2013, p. 1) como "o conjunto de atividades realizadas com o fim de utilizar, compartilhar e desenvolver o conhecimento de uma organização e dos indivíduos que nela trabalham, encaminhando-os para a melhor consecução de seus objetivos".

Destaca-se que este foco no conhecimento tem passado por um processo de implementação dentro das organizações, onde num primeiro momento o centro das atenções eram os dados, passando, num segundo momento, para a informação, e seguindo as características do ambiente em nível interno, surge, o que se tem hoje, uma valorização mais voltada ao conhecimento (CONTRERAS; HUAMANI, 2013).

Juárez e Cervantes (2012) observam que a demanda da sociedade por mais informação da Administração Pública faz com que seja necessário estabelecer novas vias de interação, que estão diretamente relacionadas ao desenvolvimento da sociedade do conhecimento, cujo compromisso deve abarcar, entre outros, os seguintes aspectos: i) a transparência e eficácia na gestão pública; ii) a produtividade nas organizações públicas; iii) a capacitação na formação dos gestores e funcionários públicos; e iv) a melhora e variedade na oferta de serviços públicos de qualidade. Desta forma, os autores afirmam que "a gestão do conhecimento resulta numa ferramenta de grande transcendência para as organizações públicas, cujo maior capital reside nas pessoas que a integram e nos conhecimentos, tanto fáticos como procedimentais".

Juárez e Cervantes (2012, p.1) destacam, ainda, que "a evolução da sociedade do conhecimento leva consigo certas mudanças sociais e econômicas que influenciam de maneira decisiva na gestão das organizações públicas, as quais assumem novas responsabilidades relacionadas com a configuração e o desenvolvimento da sociedade do conhecimento".

Pardo et al. (2013, p. 1) afirmam que "as instituições públicas são grandes produtores e consumidores de conhecimento", as quais devem prestar especial atenção a dois aspectos essenciais, quais sejam: "i) ser altamente eficiente em arrecadar e aplicar adequadamente os recursos; e ii) melhorar a capacidade de vida de seus cidadãos mediante os serviços especializados que prestam".

Assim, a gestão do conhecimento na Administração Pública refere-se ao conhecimento público dos indivíduos que a compõem, ou seja, além da própria administração, deve-se considerar, entre outros, a sociedade e os servidores, em que quando bem articulados na gestão do conhecimento farão melhor uso dos recursos e servirão de alavanca impulsionadora ao sucesso administrativo (BEM; PRADO; DELFINO, 2013).

Perspectivas em Gestão \& Conhecimento, João Pessoa, v. 9, n. 1, p. 24-41, jan./abr. 2019 
Neste contexto, essa nova sociedade e economia baseadas no conhecimento têm afetado as organizações públicas, as quais são autenticas organizações intensivas em informação, pois elas precisam adaptar sua gestão para satisfazer os novos requisitos impostos pela sociedade e pela tecnologia. Vê-se, portanto, o avanço de uma gestão transparente, com mais automatização dos processos internos, cuja introdução de técnicas de gestão do conhecimento mostra-se necessária para a formação dos gestores públicos. Isto porque a importância da gestão do conhecimento nas políticas governamentais se fundamenta numa conglomeração do conhecimento, o qual é complementado por experiências vividas (MARTÍNEZ; LARA-NAVARRA; BELTRÁN, 2006).

De igual modo, ao mesmo tempo em que as organizações valorizam a importância do conhecimento, consideram também a importância da realização de processos de controle desse recurso, mediante diferentes sistemas de mediações ou processos de auditoria. A auditoria do conhecimento é um enfoque gerencial que contribui para o fortalecimento deste recurso na organização, de forma contínua e indispensável, com vistas a permitir que a gestão tenha maior vantagem entre aquelas que não reconhecem e não impulsionam a gestão do conhecimento em todos os níveis da instituição (Dante, 2008). No entanto, na Administração Pública a gestão do capital intelectual tem se mostrado com menor esforço em sua mensuração e registro (MARTíNEZ; LARA-NAVARRA; BELTRÁN, 2006).

Desta forma, com vistas a identificar o nível de gestão do conhecimento aplicado na Administração Pública, este artigo tem como objetivo verificar a maturidade da gestão do conhecimento em uma Instituição Pública Catarinense.

\section{FUNDAMENTAÇÃO TEÓRICA}

Essa seção visa delinear os fundamentos conceituais necessários para o desenvolvimento desta pesquisa, tratando da gestão do conhecimento e maturidade, da gestão do conhecimento na Administração Pública e da avaliação da maturidade da gestão do conhecimento na Administração Pública, por meio do modelo proposto por Helou (2015).

\subsection{Gestão do Conhecimento e Maturidade}

Oliveira et al. (2011, p. 11) entendem que as definições apresentadas na literatura para a gestão do conhecimento se completam, tendo como ponto comum o processo. Neste sentido, definem os autores a gestão do conhecimento "como um conjunto de processos que visam à criação, armazenamento, disseminação e utilização do conhecimento, alinhados com os objetivos de negócio, considerando fontes de conhecimento internas e externas à organização".

Menezes et al. (2017, p. 147) definem a gestão do conhecimento como "a capacidade de lidar de forma criativa com as diferentes dimensões do conhecimento". Ribeiro et al (2017) destacam que a gestão do conhecimento contribui para maximizar o desempenho e os resultados organizacionais. No entanto, observam que o gerenciamento do conhecimento tem sido um desafio para os gestores contemporâneos, visto a dificuldade de mensurá-lo objetivamente. "A criação de estratégias adequadas e a mobilização organizacional para a ocorrência da gestão de conhecimento é o que vai diferenciar cada vez mais a competitividade entre as empresas" (RIBEIRO et al., 2017, p. 13).

Mas esse gerenciamento não é tarefa fácil. Como apontam Oliveira et al. (2011) há uma dificuldade das organizações em identificar o progresso da gestão do conhecimento, assim como avaliar os resultados obtidos com a sua adoção. Isso porque, o conhecimento é tido como um recurso intangível.

Perspectivas em Gestão \& Conhecimento, João Pessoa, v. 9, n. 1, p. 24-41, jan./abr. 2019 
Como forma de gerenciamento tem-se os modelos de avaliação da maturidade da gestão do conhecimento, os quais "permitem às organizações avaliarem sua evolução quanto a um determinado conteúdo" (OLIVEIRA et al., 2011, p. 12). Kraemer et al. (2017, p. 68) destacam que os modelos de maturidade de GC "são utilizados para superar a natureza estática das avaliações de GC e podem formalmente capturar o processo de desenvolvimento avaliando em que medida a GC é explicitamente definida, gerenciada e controlada".

Os autores Kraemer et al. $(2017$, p.68) apontam as razões pelas quais os modelos de maturidade de GC são importantes, são elas:

\begin{abstract}
Em primeiro lugar, a implementação bem sucedida de iniciativas de GC exige uma abordagem holística, sistemática e estruturada para desenvolver, medir e melhorar continuamente os processos organizacionais relacionados. Em segundo lugar, modelos de maturidade servem como uma ferramenta eficaz para facilitar a governança de GC em toda a organização. Em terceiro lugar, auxilia a identificar barreiras à implementação da GC. Em quarto lugar, modelos de maturidade podem facilitar o planejamento de curto e longo prazo.
\end{abstract}

O estudo de Kraemer et al. (2017) mostra que o campo de pesquisa da maturidade de GC é fértil, pois oferece várias possibilidades, tais como a criação de novos modelos, as mudanças de níveis de maturidade e aplicação de modelos. De igual modo, Oliveira et al. (2011) apontam existir uma lacuna em pesquisas que tratem da avalição da gestão do conhecimento no que tange a processos e resultados. É neste sentido que o presente artigo visa contribuir.

\title{
2.2 Gestão do Conhecimento na Administração Pública
}

Denhart e Catlaw (2017) observam que os desafios e oportunidades na Administração Pública estão em entender como o avanço democrático de metas públicas está ligado à administração democrática e as aspirações pessoais dos trabalhadores de instituições públicas. Nesse sentido indagam como o conhecimento pode melhorar o próprio bem-estar e o bemestar da comunidade a qual estamos inseridos. Para os autores "as pessoas, obviamente, adquirem conhecimento de maneiras diversas". No entanto, destacam que, "existe ainda uma vasta quantidade de informações que precisamos aprender e diferentes formas pelas quais podemos obtê-las" (DENHART e CATLAW, 2017, p. 3).

Os autores (Denhart e Catlaw, 2017, p. 16), ao refletirem sobre as teorias que regem a Administração pública, observam que "os profissionais da administração [pública] têm que decidir sobre os tipos de conhecimentos de que necessitam, como estes conhecimentos podem ser efetivamente obtidos e como podem ser aplicados". Nesse sentido, uma "teoria integrada da organização pública deve esclarecer e dar sentido às diferentes formas de aquisição de conhecimento nas e sobre as organizações públicas".

O estudo da gestão do conhecimento com aplicação prática na Administração Pública busca identificar aspectos que podem contribuir para uma melhor qualidade do serviço público. "A utilização da gestão do conhecimento no setor público é uma estratégia de um novo caminho para o melhor desempenho e para o melhor relacionamento interno e externo das organizações desse setor" (FRESNEDA e GONÇALVES, 2007, p. 5). Ela pode ser conceituada sob dois aspectos, sendo eles, o da política pública e o da ferramenta gerencial. O primeiro quando busca a efetividade e excelência da administração, o segundo quando visa otimizar processos e eliminar redundância, mapear e compartilhar o conhecimento organizacional (BOTELHO, 2009). Fresneda e Gonçalves (2007, p. 15) observam que:

Perspectivas em Gestão \& Conhecimento, João Pessoa, v. 9, n. 1, p. 24-41, jan./abr. 2019 
[...] a capacidade de uma nação e em especial, do setor público, para desenvolver contextos e sistemas que apoiem o compartilhamento e a criação de conhecimento na área pública visando gerar benefícios para a sociedade, aumenta em importância e deve ser objeto de maior consideração.

As razões pelas quais a Administração Pública deve se envolver com a gestão do conhecimento são explicitadas por Angelis (2011), nas quais se destacam, os ambientes diversos e turbulentos que exigem alta capacidade de adaptação e resolução de problemas; a rápida mudança em tecnologias da informação e comunicação, fazendo com que a consolidação de que o conhecimento é um insumo estratégico da produção na Economia do Conhecimento, "as competências das pessoas envolvidas na formulação e implementação de políticas públicas devem ser tratadas com as devidas ferramentas e metodologias"; e a cada vez mais a exigência da sociedade por serviços de qualidade e transparência na aplicação dos recursos, fazendo com que a administração busque novas formas de gerenciamento. $\mathrm{O}$ autor acrescenta, ainda, que, "em ambientes cada vez mais incertos, dinâmicos e complexos, tornase cada vez mais importante para a Administração Pública utilizar ferramentas mais modernas de gestão, como a gestão do conhecimento" (ANGELIS, 2011).

No entanto, a manutenção da gestão do conhecimento requer alguns esforços. Santos e Bastos (2018, p.10) apontam os desafios para a implantação da gestão do conhecimento na Administração Pública, sendo eles:

[...] a forma como as mudanças das políticas organizacionais são tratadas, a conscientização dos agentes envolvidos para que a implantação tenha resultados satisfatórios, e ainda, para que o conhecimento seja tratado como um recurso estratégico há a necessidade de se ter investimento, de modo que por vezes o custo de sua implantação torna-se também uma barreira.

Santos e Bastos (2018) destacam que as questões investigadas em gestão do conhecimento na Administração Pública centram-se em diagnósticos e implantação, sendo as avaliações de impacto após a implantação da gestão do conhecimento uma área de estudo ainda incipiente, sendo ainda um campo amplo a ser explorado.

Assim identificar o nível de maturidade em gestão do conhecimento de uma instituição pública é o primeiro passo para o aprimoramento das técnicas utilizadas, bem como dos avanços necessários para a institucionalização do conhecimento organizacional, com vistas à melhoria contínua dos processos administrativos e garantidores de melhores resultados e da qualidade dos serviços prestados.

A Figura 1 sintetiza a relação entre a Administração Pública, a Gestão do Conhecimento e a Maturidade em Gestão do Conhecimento. 
Figura 1 - Relação: Administração Pública / Gestão do Conhecimento / Maturidade GC

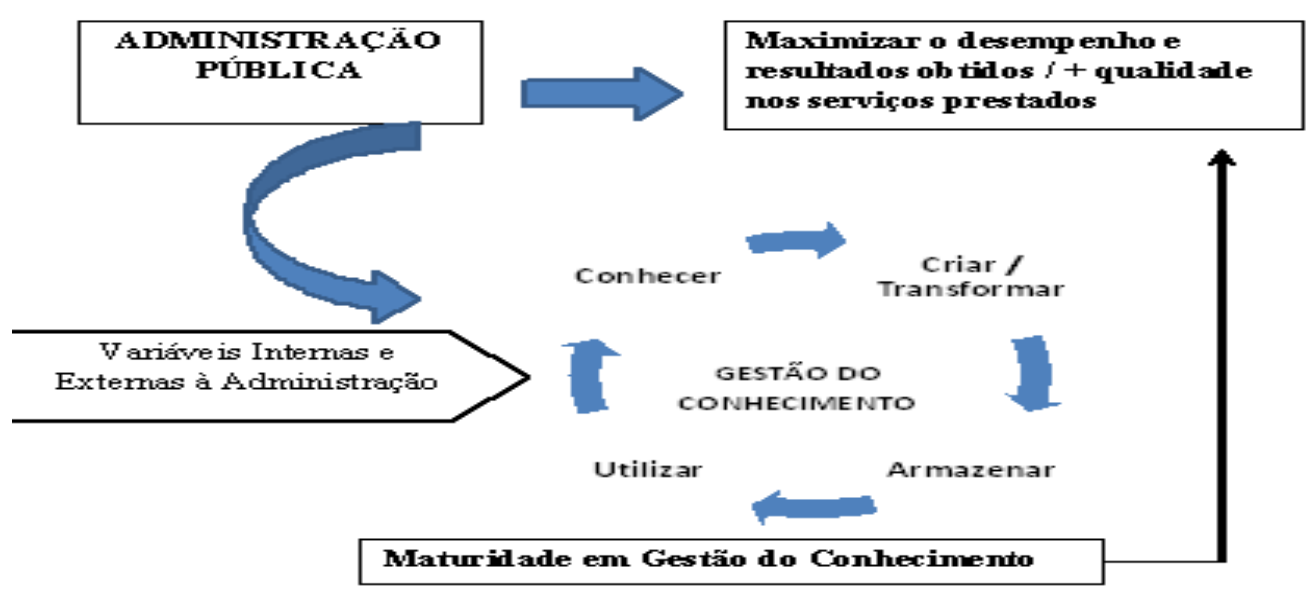

Fonte: Elaborado pelos autores

\subsection{Avaliação da Maturidade da Gestão do Conhecimento na Administração Pública: Modelo proposto por Helou (2015)}

Este estudo utiliza como fundamento de aplicação a metodologia desenvolvida por Helou (2015), a qual adaptou os modelos propostos pela APO (2009) e MGCAPB (Batista, 2012) para avaliar a maturidade da gestão do conhecimento na Administração Pública.

O modelo consiste na aplicação de um questionário que se utiliza de nove dimensões avaliativas, quais sejam, liderança; pessoas; processos; tecnologia; processo de GC; aprendizagem e inovação; resultados da GC; cultura organizacional; e arcabouço legal.

Cada dimensão é dividida em cinco categorias que devem ser pontuadas de acordo com uma escala Likert. Destaca-se que Helou (2015) manteve a escala proposta nos modelos da APO (2009) e MGCAPB (Batista, 2012), como segue:

1 - as ações descritas são muito mal realizadas, ou ainda não são realizadas.

2 - as ações descritas são mal realizadas.

3 - as ações descritas são realizadas de forma adequada.

4 - as ações descritas são bem realizadas.

5 - as ações descritas são muito bem realizadas.

\subsubsection{Avaliação das Dimensões}

Neste tópico são apresentados os critérios de avaliação, de acordo com cada dimensão, estipulados para a análise da maturidade da gestão do conhecimento em uma organização.

A dimensão liderança avalia se a iniciativa de gestão do conhecimento é impulsionada na organização; se é garantido o alinhamento da gestão do conhecimento à estratégia e aos projetos, com a missão e visão da organização; e se são fornecidos suporte e recursos para a implementação de projetos de gestão do conhecimento (APO, 2009).

Para Helou (2015, p. 137), a dimensão liderança é:

[...] reconhecimento da importância da criação e compartilhamento de conhecimento como recurso estratégico; fatores que conduzem ao estabelecimento de uma cultura e de uma arquitetura de GC; importância de alinhar as estratégias de GC às estratégias da organização; arranjos

Perspectivas em Gestão \& Conhecimento, João Pessoa, v. 9, n. 1, p. 24-41, jan./abr. 2019 
organizacionais para formalizar e elaborar a autoavaliação de GC; empoderamento das chefias intermediárias para elaboração e acompanhamento de GC.

Quadro 1 - Avaliação da dimensão liderança

\begin{tabular}{|c|c|c|c|c|c|c|}
\hline \multicolumn{7}{|c|}{ DIMENSÃO LIDERANÇA } \\
\hline Categoria & Afirmação & 1 & 2 & 3 & 4 & 5 \\
\hline $\begin{array}{l}\text { Conhecimento como } \\
\text { recurso estratégico }\end{array}$ & $\begin{array}{l}\text { A liderança reconhece a importância da criação e do } \\
\text { compartilhamento do conhecimento como recurso } \\
\text { estratégico dentro da organização. }\end{array}$ & & & & & \\
\hline $\begin{array}{l}\text { Responsabilidade e } \\
\text { trabalho colaborativo }\end{array}$ & $\begin{array}{l}\text { A liderança utiliza-se do empoderamento às chefias } \\
\text { intermediárias para realização de trabalhos colaborativos } \\
\text { e em equipe. }\end{array}$ & & & & & \\
\hline $\begin{array}{l}\text { Valorização das } \\
\text { pessoas e alocação de } \\
\text { recursos }\end{array}$ & $\begin{array}{l}\text { A liderança reconhece, promove a melhoria do } \\
\text { desempenho, a aprendizagem individual } \\
\text { compartilhamento do conhecimento garantindo a } \\
\text { alocação de recursos financeiros nos projetos de GC. }\end{array}$ & & & & & \\
\hline $\begin{array}{l}\text { Missão, visão e valores } \\
\text { organizacionais }\end{array}$ & $\begin{array}{l}\text { A liderança reconhece a importância de alinhar a GC aos } \\
\text { objetivos estratégicos da organização. }\end{array}$ & & & & & \\
\hline Maturidade de GC & $\begin{array}{l}\text { A liderança percebe a necessidade de avaliar a } \\
\text { maturidade de } \mathrm{GC} \text { nas etapas de implementar o processo } \\
\text { de GC. }\end{array}$ & & & & & \\
\hline
\end{tabular}

Fonte: Helou (2015) adaptado de APO (2009) e MGCAPB (BATISTA, 2012)

A dimensão pessoas avalia se os indivíduos são usuários, bem como geradores de conhecimento, se eles criam e processam capital intelectual dentro da organização; e se a confiança é utilizada como um pré-requisito para o compartilhamento de conhecimentos (APO, 2009).

Para Helou (2015, p. 138), a dimensão pessoas é:

[...] reconhecimento da importância do conhecimento organizacional; prontidão para autoavaliação e acompanhamento do processo de GC; sistema de incentivo para participação; existência de uma cultura de compartilhamento do conhecimento; formação de equipes de trabalho para elaboração do programa de GC; programa de capacitação para GC.

Quadro 2 - Avaliação da dimensão pessoas

\begin{tabular}{|l|l|l|l|l|l|l|}
\hline \multicolumn{7}{|c|}{ DIMENSÃO PESSOAS } \\
\hline \multicolumn{1}{|c|}{ Categoria } & \multicolumn{1}{|c|}{ Afirmação } & $\mathbf{1}$ & $\mathbf{2}$ & $\mathbf{3}$ & $\mathbf{4}$ \\
\hline $\begin{array}{l}\text { Auditoria } \\
\text { competências }\end{array}$ & $\begin{array}{l}\text { A organização conta com uma auditoria de } \\
\text { conhecimentos para alimentar permanentemente o } \\
\text { banco de competências dos seus servidores. }\end{array}$ & & & \\
\hline Capacitação & $\begin{array}{l}\text { Existe algum programa de capacitação dos } \\
\text { colaboradores para reconhecer o conhecimento como } \\
\text { recurso organizacional de maneira a contribuir no } \\
\text { projeto de GC. }\end{array}$ & & & \\
\hline
\end{tabular}




\begin{tabular}{|l|l|l|l|l|l|}
\hline $\begin{array}{l}\text { Trabalho compartilhado } \\
\text { e colaborativo }\end{array}$ & $\begin{array}{l}\text { A organização do trabalho contempla a formação de } \\
\text { equipes que suportam o trabalho compartilhado e } \\
\text { colaborativo. }\end{array}$ & & & & \\
\hline $\begin{array}{l}\text { Conhecimento para } \\
\text { tomada de decisão }\end{array}$ & $\begin{array}{l}\text { Os colaboradores reconhecem a importância do recurso } \\
\text { conhecimento como elemento de tomada de decisão. }\end{array}$ & & & \\
\hline $\begin{array}{l}\text { Incentivo para } \\
\text { participação } \\
\text { compartilhamento }\end{array}$ & $\begin{array}{l}\text { Existe algum sistema de incentivo para encorajar a } \\
\text { participação dos colaboradores no projeto de GC. }\end{array}$ & & & \\
\hline
\end{tabular}

Fonte: Helou (2015) adaptado de APO (2009) e MGCAPB (BATISTA, 2012)

A dimensão processos avalia se há medidas sociais e tecnológicas que aumentam a contribuição do conhecimento na organização; se há processos sistemáticos e eficazes que podem contribuir para a melhoria organizacional, por meio da produtividade, rentabilidade, qualidade e crescimento. Esta dimensão refere-se ao desenvolvimento do conhecimento e dos processos de conversão, os quais utilizam cinco passos, quais sejam, identificar; criar; armazenar; compartilhar; e aplicar (APO, 2009).

Para Helou (2015, p. 138) a dimensão processos é:

[...] definição das competências alinhadas à missão e ao objetivo da organização; mapeamento e gerenciamento dos processos organizacionais; avaliação contínua do processo; conhecimento para execução dos processos organizacionais; formalização do processo de coleta e compartilhamento do conhecimento; existência de um sistema de GC.

Quadro 3 - Avaliação da dimensão processos

\begin{tabular}{|l|l|l|l|l|l|}
\hline \multicolumn{1}{|c|}{ DIMENSÃO PROCESSOS } & $\mathbf{1}$ & $\mathbf{2}$ & $\mathbf{3}$ & $\mathbf{4}$ & $\mathbf{5}$ \\
\hline \multicolumn{1}{|c|}{ Categoria } & \multicolumn{1}{|c|}{ Afirmação } & & & \\
\hline Conhecimentos essenciais. & $\begin{array}{l}\text { Os conhecimentos essenciais requeridos na execução } \\
\text { dos processos organizacionais já são conhecidos da } \\
\text { organização. }\end{array}$ & & & & \\
\hline $\begin{array}{l}\text { Alinhamento de GC ao } \\
\text { planejamento estratégico. }\end{array}$ & $\begin{array}{l}\text { A organização define seus conhecimentos essenciais } \\
\text { e os alinha à sua missão e aos objetivos da } \\
\text { organização. }\end{array}$ & & & & \\
\hline $\begin{array}{l}\text { Coleta e compartilhamento } \\
\text { de conhecimento. }\end{array}$ & $\begin{array}{l}\text { O processo de coletar e compartilhar conhecimentos } \\
\text { é sistematizado dentro da organização. }\end{array}$ & & & \\
\hline Avaliação por indicadores. & $\begin{array}{l}\text { Os processos organizacionais são mapeados e } \\
\text { gerenciados a partir de indicadores de desempenho. }\end{array}$ & & & & \\
\hline Foco nos resultados. & $\begin{array}{l}\text { A organização avalia e melhora continuamente seus } \\
\text { processos para alcançar melhor desempenho, } \\
\text { eficiência, eficácia e efetividade. }\end{array}$ & & & \\
\hline
\end{tabular}

Fonte: Helou (2015) adaptado de APO (2009) e MGCAPB (BATISTA, 2012).

A dimensão tecnologia avalia se é acelerado o processo de conhecimento por meio de ferramentas de tecnologia eficazes; se as ferramentas, como groupware e espaços de trabalho colaborativos, permitem a participação ao longo do tempo e a distância; e se é fornecida uma plataforma para a retenção do conhecimento organizacional (APO, 2009). 
Para Helou (2015, p. 138), a dimensão tecnologia é "sistema de TI para suportar unidades da organização; alinhamento da $\mathrm{TI}$ com as estratégias organizacionais; disponibilização das informações; apropriação das TI aos usuários; TI para comunicação para transferência e compartilhamento de conhecimento; TI para suportar processo GC".

Quadro 4 - Avaliação da dimensão tecnologia

\begin{tabular}{|c|c|c|c|c|c|c|}
\hline \multicolumn{7}{|c|}{ DIMENSÃO TECNOLOGIA } \\
\hline Categoria & Afirmação & 1 & 2 & 3 & 4 & 5 \\
\hline $\begin{array}{ll}\text { Tecnologia } & \text { na } \\
\text { modelagem } & \text { de } \\
\text { processos. } & \end{array}$ & $\begin{array}{l}\text { A organização modela seus sistemas de trabalho } \\
\text { incluindo novas tecnologias e o compartilhamento do } \\
\text { conhecimento. }\end{array}$ & & & & & \\
\hline Eficácia tecnológica. & $\begin{array}{l}\text { A infraestrutura de TI disponível é suficiente para } \\
\text { suportar as estratégias da organização e às } \\
\text { necessidades dos usuários. }\end{array}$ & & & & & \\
\hline $\begin{array}{l}\text { Tecnologia como meio de } \\
\text { compartilhamento. }\end{array}$ & $\begin{array}{l}\text { A tecnologia é usada como fonte de comunicação e } \\
\text { como apoio à transferência e ao compartilhamento de } \\
\text { conhecimento. }\end{array}$ & & & & & \\
\hline $\begin{array}{l}\text { Tecnologia como meio de } \\
\text { conhecimento. }\end{array}$ & $\begin{array}{l}\text { Os dados, informações e conhecimentos disponíveis na } \\
\text { organização são regularmente atualizados. }\end{array}$ & & & & & \\
\hline $\begin{array}{l}\text { Tecnologia como meio de } \\
\text { recuperação de } \\
\text { conhecimentos. }\end{array}$ & $\begin{array}{l}\text { A tecnologia disponível já permite arranjar, tornar } \\
\text { acessível, proteger, armazenar, recuperar, analisar, } \\
\text { filtrar, avaliar e dispor sobre os dados, informações e } \\
\text { conhecimentos relevantes para a organização. }\end{array}$ & & & & & \\
\hline
\end{tabular}

Fonte: Helou (2015) adaptado de APO (2009) e MGCAPB (BATISTA, 2012)

A dimensão processo de gestão do conhecimento avalia se a organização tem processo sistemático voltado à gestão do conhecimento, se há um mapa de conhecimento formulado na organização; se são observadas as fases do conhecimento (APO, 2009).

Quadro 5 - Avaliação da dimensão processo de gestão do conhecimento

\begin{tabular}{|c|c|c|c|c|c|c|}
\hline \multicolumn{7}{|c|}{ DIMENSÃO PROCESSO de GC } \\
\hline Categoria & Afirmação & 1 & 2 & 3 & 4 & 5 \\
\hline Processos de GC. & $\begin{array}{l}\text { A organização tem processos sistemáticos de } \\
\text { identificação, } \\
\text { compartilhamento e utilização, do conhecimento. }\end{array}$ & & & & & \\
\hline Mapa de conhecimento. & $\begin{array}{l}\text { A organização conta com um mapa de conhecimento e } \\
\text { distribui os ativos ou recursos de conhecimento por } \\
\text { toda a organização. }\end{array}$ & & & & & \\
\hline $\begin{array}{l}\text { Processo de aquisição e } \\
\text { armazenamento do } \\
\text { conhecimento. }\end{array}$ & $\begin{array}{l}\text { O conhecimento adquirido após a execução de tarefas } \\
\text { e a conclusão de projetos é registrado e compartilhado. }\end{array}$ & & & & & \\
\hline $\begin{array}{ll}\text { Retenção } & \text { do } \\
\text { conhecimento } & \text { na } \\
\text { organização. } & \end{array}$ & $\begin{array}{l}\text { O conhecimento essencial de servidores públicos que } \\
\text { estão saindo a organização é retido. }\end{array}$ & & & & & \\
\hline $\begin{array}{l}\text { Práticas } \\
\text { aprendizagem. }\end{array}$ & $\begin{array}{l}\text { As atividades de benchmarking são realizadas dentro e } \\
\text { fora da organização, os resultados são usados para }\end{array}$ & & & & & \\
\hline
\end{tabular}

Perspectivas em Gestão \& Conhecimento, João Pessoa, v. 9, n. 1, p. 24-41, jan./abr. 2019 


\begin{tabular}{|l|l|l|l|l|l|}
\hline & $\begin{array}{l}\text { melhorar o desempenho organizacional e criar novo } \\
\text { conhecimento. }\end{array}$ & & & & \\
\hline
\end{tabular}

Fonte: Helou (2015) adaptado de APO (2009) e MGCAPB (BATISTA, 2012)

A dimensão aprendizagem e inovação avalia se o processo de conhecimento permite a aprendizagem e inovação em todos os níveis e áreas da organização; se o processo de conhecimento é considerado para novos produtos, serviços, processos, mercados, tecnologias e modelos de negócios (APO, 2009).

Quadro 6 - Avaliação da dimensão aprendizagem e inovação

\begin{tabular}{|c|c|c|c|c|c|c|}
\hline \multicolumn{7}{|c|}{ DIMENSÃO APRENDIZAGEM E INOVAÇÃO } \\
\hline Categoria & Afirmação & 1 & 2 & 3 & 4 & 5 \\
\hline $\begin{array}{l}\text { Aprendizagem } \\
\text { contínua. }\end{array}$ & $\begin{array}{l}\text { A organização articula e reforça continuamente valores como } \\
\text { a aprendizagem e a inovação. }\end{array}$ & & & & & \\
\hline $\begin{array}{l}\text { Aprendizagem a } \\
\text { partir de erros. }\end{array}$ & $\begin{array}{l}\text { A organização considera a atitude de assumir riscos ou a fato } \\
\text { de cometer erros como oportunidades de aprendizagem } \\
\text { desde que isso não ocorra repetidamente. }\end{array}$ & & & & & \\
\hline $\begin{array}{l}\text { Trabalhos em } \\
\text { interfuncionais. }\end{array}$ & $\begin{array}{l}\text { Equipes interfuncionais são formadas para resolver } \\
\text { problemas ou lidar com situações preocupantes que ocorrem } \\
\text { em diferentes unidades gerenciais da organização. }\end{array}$ & & & & & \\
\hline $\begin{array}{l}\text { Autonomia } \\
\text { funcional. }\end{array}$ & $\begin{array}{l}\text { As pessoas sentem que recebem autonomia dos seus } \\
\text { superiores hierárquicos e que suas ideias e contribuições são } \\
\text { geralmente valorizadas pela organização. }\end{array}$ & & & & & \\
\hline $\begin{array}{l}\text { Inovação na gestão } \\
\text { de pessoas. }\end{array}$ & $\begin{array}{l}\text { As chefias intermediárias estão dispostas a usar novas } \\
\text { ferramentas e métodos e estimular o trabalho em grupo com } \\
\text { o objetivo de compartilhar conhecimento. }\end{array}$ & & & & & \\
\hline
\end{tabular}

Fonte: Helou (2015) adaptado de APO (2009) e MGCAPB (BATISTA, 2012)

A dimensão resultados da gestão do conhecimento avalia o histórico da organização na implantação/implementação da gestão do conhecimento e se são utilizados indicadores para avaliar as contribuições da gestão do conhecimento nos resultados da organização (APO, 2009).

Quadro 7 - Avaliação da dimensão resultados da gestão do conhecimento

\begin{tabular}{|l|l|l|l|l|l|l|}
\hline \multicolumn{2}{|c|}{ DIMENSÃO RESULTADOS DA GC } \\
\hline \multicolumn{1}{|c|}{ Categoria } & \multicolumn{1}{|c|}{ Afirmação } & $\mathbf{1}$ & $\mathbf{2}$ & $\mathbf{3}$ & $\mathbf{4}$ & $\mathbf{5}$ \\
\hline $\begin{array}{l}\text { Histórico de GC na } \\
\text { organização. }\end{array}$ & $\begin{array}{l}\text { A organização tem um histórico de sucesso na } \\
\text { implementação da GC e de outras iniciativas de } \\
\text { mudança que pode ser comprovado com resultados de } \\
\text { indicadores de desempenho. }\end{array}$ & & & \\
\hline $\begin{array}{l}\text { Utilização de indicadores } \\
\text { para avaliação de } \\
\text { resultados. }\end{array}$ & $\begin{array}{l}\text { São utilizados indicadores para avaliar o impacto das } \\
\text { contribuições e das iniciativas de GC nos resultados da } \\
\text { organização. }\end{array}$ & & & \\
\hline $\begin{array}{l}\text { Melhoria } \\
\text { indicadores. }\end{array}$ & $\begin{array}{l}\text { A organização melhorou - graças às contribuições e às } \\
\text { iniciativas da GC - os resultados relativos aos } \\
\text { indicadores de qualidade dos produtos e serviços e }\end{array}$ & & & \\
\hline
\end{tabular}

Perspectivas em Gestão \& Conhecimento, João Pessoa, v. 9, n. 1, p. 24-41, jan./abr. 2019 


\begin{tabular}{|l|l|l|l|l|}
\hline & eficiência. & & & \\
\hline $\begin{array}{l}\text { Indicadores de } \\
\text { efetividade social. }\end{array}$ & $\begin{array}{l}\text { A organização melhorou - graças às contribuições e às } \\
\text { iniciativas de GC - os resultados relativos aos } \\
\text { indicadores de efetividade social. }\end{array}$ & & & \\
\hline $\begin{array}{l}\text { Indicadores dos critérios } \\
\text { gerais da Administração } \\
\text { Pública brasileira. }\end{array}$ & $\begin{array}{l}\text { A organização melhorou - graças às contribuições e às } \\
\text { iniciativas de GC - os resultados dos indicadores de } \\
\text { legalidade, impessoalidade, publicidade, moralidade e } \\
\text { desenvolvimento. }\end{array}$ & & & \\
\hline
\end{tabular}

Fonte: Helou (2015) adaptado de APO (2009) e MGCAPB (BATISTA, 2012)

A cultura organizacional é tida para Helou (2015, p. 213) como "todo aquele complexo que inclui conhecimento, a arte, as crenças, a lei, a moral, os costumes e todos os hábitos e aptidões adquiridos pelo homem, não somente em família, como também por fazer parte de uma sociedade, ou organização como membro dela que é".

Quadro 8 - Avalição da dimensão cultura organizacional

\begin{tabular}{|c|c|c|c|c|c|c|}
\hline \multicolumn{7}{|c|}{ DIMENSÃO CULTURA ORGANIZACIONAL } \\
\hline Categoria & Afirmação & 1 & 2 & 3 & 4 & 5 \\
\hline $\begin{array}{l}\text { Necessidade de se adotar } \\
\text { GC }\end{array}$ & $\begin{array}{l}\text { A liderança identifica as questões, os fatores e } \\
\text { elementos de sucesso que conduzem à organização ao } \\
\text { estabelecimento de uma cultura de } \mathrm{GC} \text { e de uma } \\
\text { arquitetura de GC. }\end{array}$ & & & & & \\
\hline $\begin{array}{lr}\text { Trabalhor } & \text { colaborativo } \\
\text { entre } & \text { equipes } \\
\text { interdisciplinares }\end{array}$ & $\begin{array}{l}\text { Existe uma cultura de trabalho que estimula o trabalho } \\
\text { colaborativo e em equipes interdisciplinares. }\end{array}$ & & & & & \\
\hline $\begin{array}{l}\text { Clima } \quad \text { e } \quad \text { cultura } \\
\text { organizacional }\end{array}$ & $\begin{array}{l}\text { A organização possui um clima organizacional propício } \\
\text { para a participação e para o compartilhamento de } \\
\text { conhecimentos }\end{array}$ & & & & & \\
\hline $\begin{array}{ll}\text { Cultura } & \text { de } \\
\text { compartilhamento } & \end{array}$ & $\begin{array}{l}\text { A organização compartilha as melhores práticas e } \\
\text { lições aprendidas por toda a organização para que não } \\
\text { haja um constante "reinventar da roda" e retrabalho. }\end{array}$ & & & & & \\
\hline $\begin{array}{l}\text { Processo de tomada de } \\
\text { decisão }\end{array}$ & $\begin{array}{l}\text { Existe uma cultura de participação no processo de } \\
\text { tomada de decisão }\end{array}$ & & & & & \\
\hline
\end{tabular}

Fonte: Helou (2015) adaptado de APO (2009) e MGCAPB (BATISTA, 2012)

De acordo com Helou (2015, p. 159), arcabouço legal é "um atributo jurídico de qualquer ato humano ou pessoa jurídica que indica se é, ou não, contrário à lei, se está, ou não, dentro do permitido pelo sistema jurídico, seja expressamente ou implicitamente. Se este atributo for positivo, diz-se que é legal, caso contrário é ilegal".

Quadro 9 - Avaliação da dimensão arcabouço legal

\begin{tabular}{|c|c|c|c|c|c|c|}
\hline \multicolumn{7}{|c|}{ DIMENSÃO ARCABOUÇO LEGAL } \\
\hline Categoria & Afirmação & 1 & 2 & 3 & 4 & 5 \\
\hline Orçamento. & $\begin{array}{l}\text { Existe item orçamentário para garantir a implantação das } \\
\text { iniciativas de GC. }\end{array}$ & & & & & \\
\hline Legalidade. & Existe uma articulação entre os organismos institucionais & & & & & \\
\hline
\end{tabular}

Perspectivas em Gestão \& Conhecimento, João Pessoa, v. 9, n. 1, p. 24-41, jan./abr. 2019 


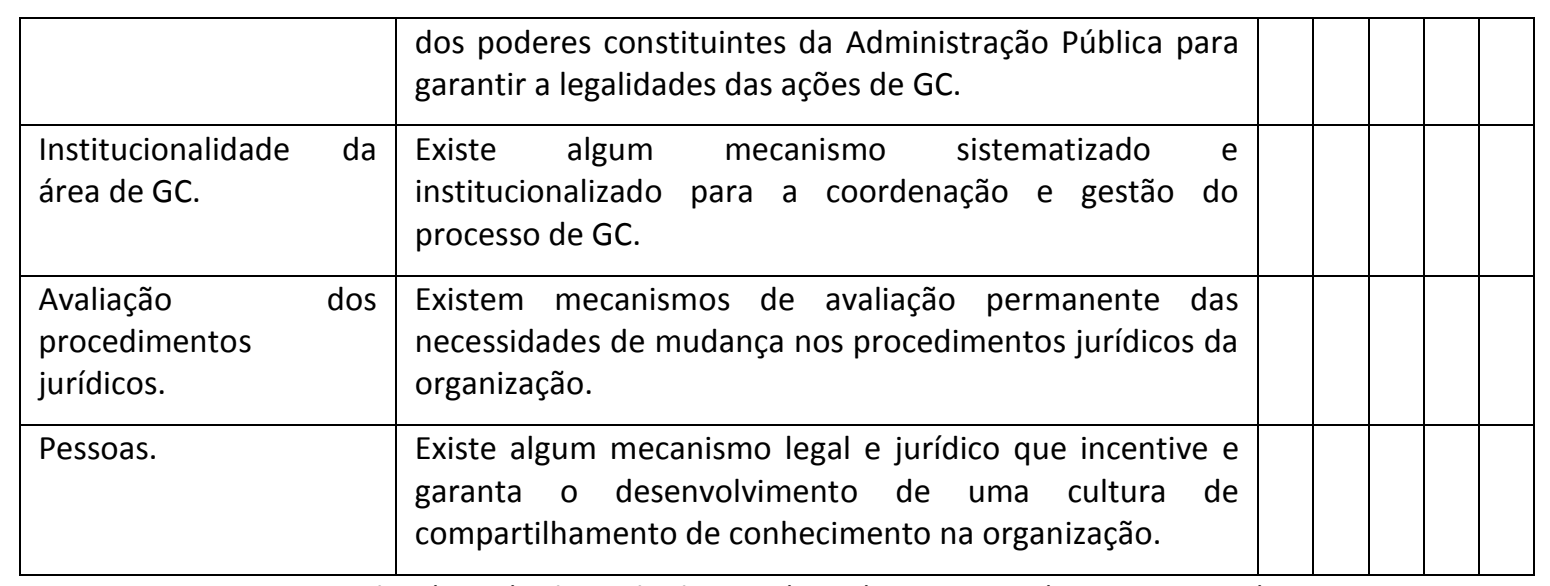

Fonte: Helou (2015) adaptado de APO (2009) e MGCAPB (BATISTA, 2012).

Com base na pontuação total obtida é possível identificar o nível de maturidade na gestão do conhecimento da organização (APO, 2009; Batista, 2012; Helou, 2015).

\subsubsection{Níveis de Maturidade em GC}

Os resultados da auditoria dão uma visão do nível de amadurecimento da gestão de conhecimento em uma organização. Isso pode variar a partir do nível "reação" no seu nível mais baixo, até o nível de "maturidade" no seu nível mais elevado (APO, 2009). A APO (2009) estabelece cinco níveis do menor para o maior, quais sejam: i) reação; ii) iniciação; iii) introdução / expansão; iv) refinamento / controle; e v) maturidade.

Helou (2015) renomeou os níveis, uma vez que incluiu duas novas dimensões avaliativas, cultura organizacional e arcabouço legal, nos modelos propostos pela APO (2009) e Batista (2012). Desta forma, para Helou (2015), a escala de maturidade em uma organização da Administração Pública baseia-se em $5 C^{\prime}$ s, são eles: i) carente; ii) constatando; iii) consciente; iv) consistente; e v) consolidado.

Considerando que da análise das dimensões pode-se obter a pontuação máxima de 225 pontos, tem-se que a maturidade de uma organização pode ser evidenciada conforme exposto na Figura 1 - Nível de Maturidade em Gestão do Conhecimento.

Figura 2 - Nível de Maturidade em Gestão do Conhecimento

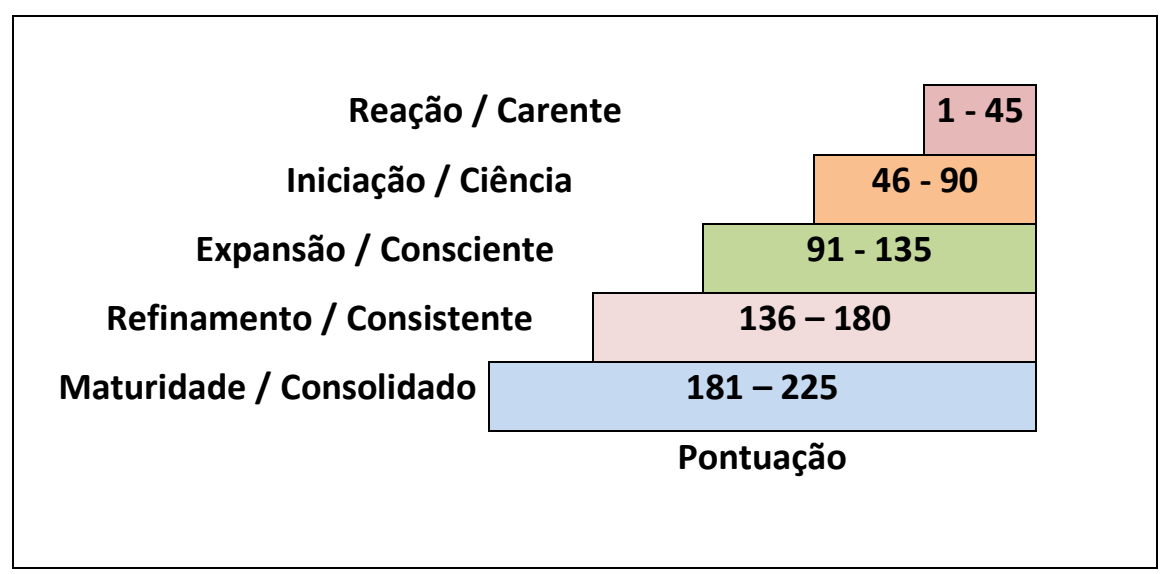

Fonte: Elaborado pelos autores a partir de APO, 2009; Batista, 2012; Helou, 2015

O significado de cada um dos cinco níveis é definido como segue (APO, 2009; Batista, 2012; Helou, 2015):

Perspectivas em Gestão \& Conhecimento, João Pessoa, v. 9, n. 1, p. 24-41, jan./abr. 2019 
- Nível 1 - Reação / Carente: A organização não tem conhecimento de que é gestão do conhecimento e qual a sua importância para melhorar a eficiência, eficácia e efetividade de suas ações.

- Nível 2 - Iniciação / Ciência: A organização está começando a reconhecer a necessidade de gerir o conhecimento ou pode já estar iniciando um projeto piloto de gestão do conhecimento. Há práticas de gestão do conhecimento em algumas áreas.

- Nível 3 - Expansão / Consciente: Há práticas de gestão do conhecimento em algumas áreas. Há a iniciativa de gestão do conhecimento de maneira isolada.

- Nível 4 - Refinamento / Consistente: A implementação da GC é continuamente avaliada para a melhoria contínua. Verifica-se o início do processo de gestão do conhecimento de modo integrado.

- Nível 5: Maturidade / Consolidado: O processo de gestão do conhecimento encontra-se institucionalizado dentro da organização pública.

De igual modo, aplicam-se os níveis de maturidade de forma intermediária às dimensões, como demonstrado na Figura 2 - Nível Intermediário de Maturidade por Dimensão.

Figura 3 - Nível Intermediário de Maturidade por Dimensão

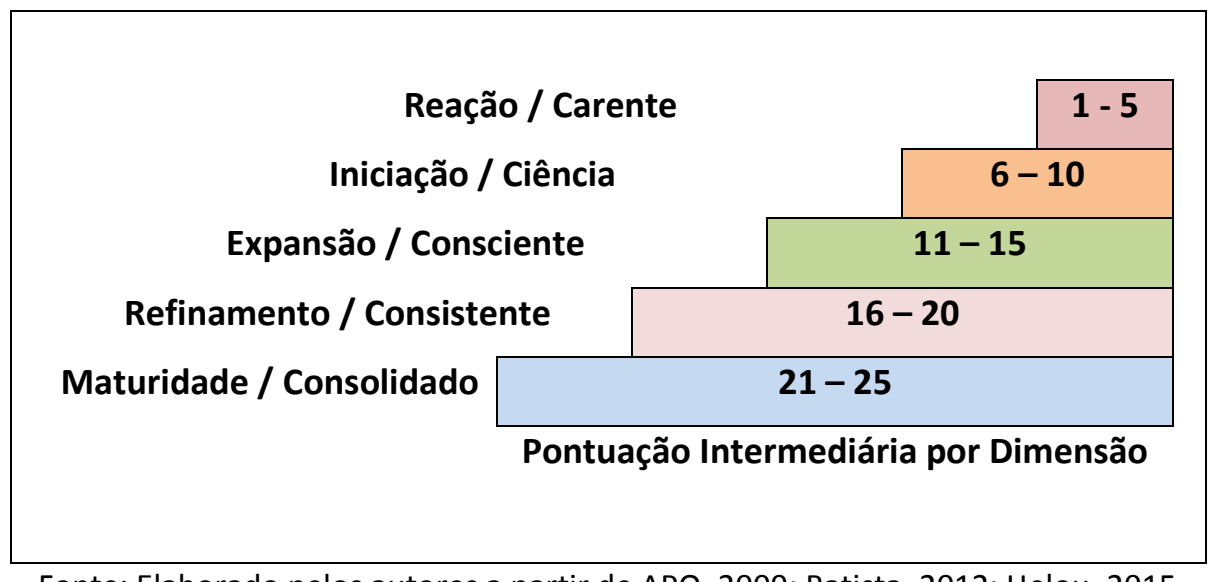

Fonte: Elaborado pelos autores a partir de APO, 2009; Batista, 2012; Helou, 2015

\section{DIRETRIZES METODOLÓGICAS}

Definiu-se pela aplicação do modelo proposto por Helou (2015), o qual visa avaliar a maturidade da gestão do conhecimento na Administração Pública. Trata-se de uma pesquisa de abordagem qualitativa de cunho exploratório descritivo, na qual se delimitou a aplicação do modelo em uma única organização pública do Estado de Santa Catarina - Brasil, tendo como pergunta de pesquisa: qual o nível de maturidade em gestão do conhecimento que a organização se encontra?

A coleta de dados foi realizada em maio de 2016, por meio dos questionários que compõem o modelo, explicitados no item 2.2.1 deste artigo, os quais foram aplicados a um coordenador de setor da instituição investigada, a fim de identificar qual o grau de maturidade de gestão do conhecimento.

Nesse sentido, a pesquisa é um estudo de caso, uma vez que se utiliza de uma única instituição investigada, à luz do que explica Severino (2007, p. 121), "pesquisa que se concentra no estudo de um caso particular, considerado representativo de um conjunto de casos análogos, por ele significativamente representativo".

Gil (2010, p. 124) destaca que: 


\begin{abstract}
A credibilidade de um estudo de caso tem muito a ver com a adequação de seus resultados aos pontos de vista de seus participantes. De fato, os pesquisados são capazes de conhecer mais que o pesquisador acerca da realidade que está sendo estudada. Logo, eles podem atuar como avaliadores dos resultados da pesquisa.
\end{abstract}

Nesse sentido, os dados coletados foram descritos pelas dimensões apresentadas pelo modelo aplicado (Helou, 2015), quais sejam, liderança; pessoas; processos; tecnologia; processo de GC; aprendizagem e inovação; resultados da GC; cultura organizacional; e arcabouço legal, e foram codificados na análise avaliativa dos resultados. Os resultados foram apresentados ao participante da pesquisa, antes da conclusão da análise, a fim de se obter o feedback das percepções entre o resultado alcançado pela pesquisa e a realidade fática, dando assim credibilidade à qualidade dos dados e resultados obtidos.

\title{
4 RESULTADOS
}

Os resultados obtidos apontam que a instituição pública avaliada enquadra-se no nível de expansão, com 102 pontos no total, ou seja, ela tem consciência da gestão do conhecimento na organização. Entretanto, ainda é um processo incipiente, uma vez que se observa haver práticas de gestão do conhecimento em algumas áreas de forma isolada, como pode-se inferir das pontuações intermediárias obtidas em cada dimensão. O Gráfico 1 Maturidade da Gestão do Conhecimento - mostra os resultados por dimensão.

Gráfico 1 - Maturidade da Gestão do Conhecimento.

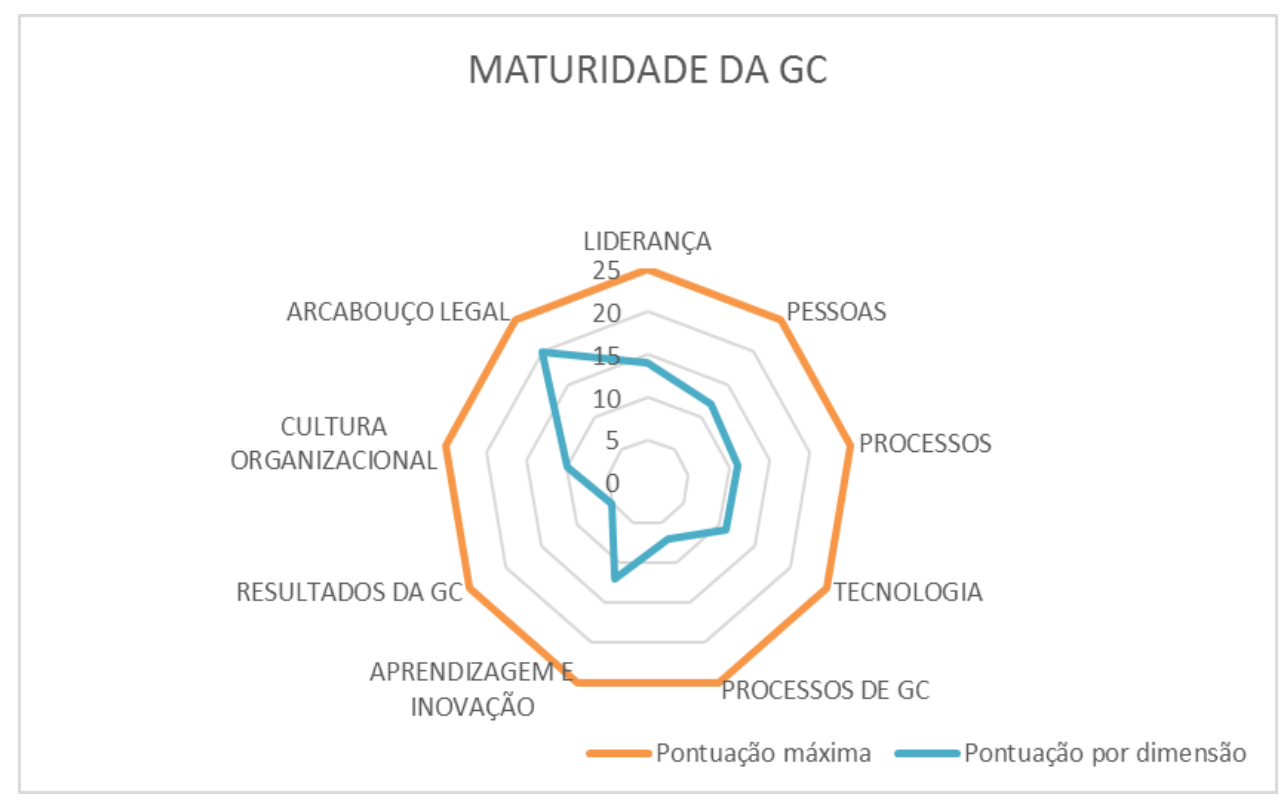

Fonte: Os autores a partir dos dados coletados

Dos resultados obtidos, percebe-se que a dimensão liderança, com quatorze pontos, está em fase de expansão. Isto se dá por algumas ações isoladas que buscam alinhar a gestão do conhecimento em ações estratégicas e de projetos voltados à missão e à visão da organização. Verifica-se que há ações iniciais de implementação da gestão do conhecimento.

A dimensão pessoas, com quatorze pontos, também se encontra em fase de expansão, ainda não se percebe de forma contumaz a importância do conhecimento organizacional. Não

Perspectivas em Gestão \& Conhecimento, João Pessoa, v. 9, n. 1, p. 24-41, jan./abr. 2019 
há processos de gestão do conhecimento formalmente constituídos, os quais estimulem uma cultura de compartilhamento do conhecimento. Verificam-se ações isoladas em programas de capacitação.

A dimensão processos apresentou onze pontos, enquadrando-se no nível de expansão. Assim, há por parte da instituição a consciência da gestão do conhecimento, sem, contudo, têla implantada por completo. Não se verificam processos sistemáticos de criação, armazenamento e compartilhamento do conhecimento com o objetivo de melhoria na qualidade dos serviços prestados.

A dimensão tecnologia, com onze pontos, também está na fase de expansão. Embora haja tecnologia da informação aplicada às atividades da instituição, não se constata a sistematização das informações de forma integrada a um conceito voltado à gestão do conhecimento.

A dimensão processo de GC totalizou sete pontos, ficando em nível de iniciação, ou seja, está se começando a conhecer a necessidade de gerir o conhecimento. Não há processo sistematizado de gestão do conhecimento, em sua totalidade, há apenas em algumas áreas.

A dimensão aprendizagem e inovação está em fase de expansão, com doze pontos. Isso significa que ainda não há, em sua completude, um processo de conhecimento que permita a aprendizagem e a inovação em todos os níveis e áreas da organização.

A dimensão resultados de GC apresentou fase de carência ou reação, atingindo somente cinco pontos. Infere-se, portanto, que não há na instituição avaliada um histórico de implantação de gestão do conhecimento, tampouco são utilizados indicadores que possam mensurar a contribuição da gestão do conhecimento nos resultados e desempenho da organização.

A dimensão cultura organizacional enquadra-se na fase de iniciação, com dez pontos, ou seja, a organização está começando a reconhecer a importância da gestão do conhecimento.

A dimensão arcabouço legal foi a que obteve maior pontuação, a qual se enquadra na fase de refinamento, com vinte pontos. É a dimensão cuja gestão do conhecimento se consolidou, tendo assumido sua maturidade dentro do processo.

\section{CONCLUSÃO}

A pergunta de pesquisa buscava responder qual o nível de maturidade em gestão do conhecimento que a organização se encontra. Utilizando-se das dimensões avaliativas propostas no modelo utilizado, quais sejam liderança, pessoas, processos, tecnologia, processo de GC, aprendizagem e inovação, resultados da GC, cultura organizacional e arcabouço legal, foi possível identificar pelos resultados obtidos que a instituição pública avaliada enquadra-se no nível de expansão, com 102 pontos no total, ou seja, ela tem consciência da gestão do conhecimento na organização. Entretanto, ainda é um processo incipiente, uma vez que se observa haver práticas de gestão do conhecimento em algumas áreas de forma isolada, como se pode inferir das pontuações intermediárias obtidas em cada dimensão.

A exemplo do que descreve Martínez; Lara-Navarra e Beltrán (2006), a incorporação de uma gestão do conhecimento nas instituições públicas permite que as informações, bem como o conhecimento, sejam considerados como um ativo da gestão administrativa, na qual se fomenta a produção de um novo conhecimento organizacional, garantindo melhores resultados.

A importância de se medir o nível em que se encontra a gestão do conhecimento em uma organização se dá pelo constante aprimoramento no processo de identificar, criar, armazenar, compartilhar e aplicar o conhecimento organizacional, de modo que a memória

Perspectivas em Gestão \& Conhecimento, João Pessoa, v. 9, n. 1, p. 24-41, jan./abr. 2019 
organizacional não se perca ao longo do tempo ou mesmo que seja desprezada pela introdução de novos conceitos e procedimentos.

Identificar o nível de maturidade em gestão do conhecimento de uma instituição pública é o primeiro passo para o aprimoramento das técnicas utilizadas, bem como dos avanços necessários para a institucionalização do conhecimento organizacional, com vistas à melhoria contínua dos processos administrativos e garantidores de melhores resultados e da qualidade dos serviços prestadores.

Aponta-se que os estudos em gestão do conhecimento na Administração Pública concentram-se em diagnósticos e implantação, sendo as avaliações de impacto após a sua implantação um campo amplo ainda a ser explorado.

\section{REFERÊNCIAS}

ANGELIS, Cristiano Trindade de. Gestão do Conhecimento no setor público: um estudo de caso por meio do método OKA. Revista do Serviço Público, Brasília, v. 62, n. 2, p. 137-166, abr./jun. 2011. Disponível em: https://revista.enap.gov.br/index.php/RSP/article/view/66. Acesso em: 07 set. 2018.

APO (Asian Productivity Organization). Knowledge Management: Facilitators' Guide. Asian Productivity Organization. 2009. Disponível em: http://www.apo-tokyo.org/00e-books/IS39 APO-KM-FG/IS-39 APO-KM-FG.pdf. Acesso em: 29 set. 2016.

BATISTA, F.F. Modelo de Gestão do Conhecimento para a Administração Pública Brasileira. Brasília: Instituto de Pesquisa Econômica Aplicada (IPEA). 2012. Disponível em: http://www.enabrasil.sc.gov.br/uploads/livro modelodegestao vol-01.pdf. Acesso em: 29 set. 2016.

BEM, Roberta Moraes; PRADO Maria Lourdes; DELFINO, Nelson. Desafios à implantação da gestão do conhecimento: a questão cultural nas organizações pública federais brasileiras. Revista Digital de Biblioteconomia e Ciência da Informação. 2013. Disponível em: http://periodicos.sbu.unicamp.br/ojs/index.php/rdbci/article/view/1641. Acesso em: 29 set. 2016.

BOTELHO, Cássia Regina Ossipe Martins. Gestão do Conhecimento para a melhoria da Gestão Pública: os caminhos da implantação da gestão do conhecimento na Câmara dos Deputados. In: CONGRESSO CONSAD DE GESTÃO PÚBLICA, 2., 2009. Painel 20: Gestão do conhecimento e inovação para a melhoria da gestão pública. 2009. Disponível em: http://consad.org.br/evento/ii-congresso/. Acesso em: 07 set. 2018.

CONTRERAS, Fortunato Contreras; HUAMANI, Pedro Leonardo. La gestión del conocimiento y las políticas públicas. Lima - Peru: Universidad Maria Auxiliadora, 2013. Disponível em: http://eprints.rclis.org/22933/1/LA\%20GESTI\%C3\%93N\%20DEL\%20CONOCIMIENTO\%20Y\%20L AS\%20POL\%C3\%8DTICAS\%20P\%C3\%9ABLICAS.pdf. Acesso em: 29 set. 2016.

DANTE, G. P. Auditoria da Informação e do Conhecimento Organizacional: Gênese de uma Integração. Brazilian Jounal of Information Science, v. 2, n.2, 2008, p.3-16. Disponível em: http://basessibi.c3sl.ufpr.br/brapci/index.php/article/view/0000008764/bb1f8f46eb747f09c4 d8907207e9efce. Acesso em: 1을 2017. 
DENHARDT, Robert B.; CATLAW, Thomas J. Teorias da Administração Pública. 2. ed. Tradução: Noveritis do Brasil; revisão técnica: Luiz Fernando Abrucio. São Paulo: Cengage Learning, 2017.

FRESNEDA, Paulo Sérgio Vilches; GONÇALVES, Sonia Maria Goulart. A Experiência Brasileira na Formulação de uma Proposta de Política de Gestão do Conhecimento para a Administração Pública Federal. Câmara dos Deputados. Centro de Documentação e Informação: Coordenação de Publicação: Brasília, 2007. Disponível em: http://www.bibl.ita.br/PoliticaGC.pdf. Acesso em: 07 set. 2018.

GIL, Antonio Carlos. Como elaborar projetos de pesquisa. 5. ed. São Paulo: Atlas, 2010.

HELOU, A. R. H. A. Avaliação da maturidade da gestão do conhecimento na Administração Pública. Tese (Doutorado em Engenharia e Gestão do Conhecimento) - Universidade Federal de Santa Catarina, Florianópolis, Brasil). 2015. Disponível em: http://btd.egc.ufsc.br/wpcontent/uploads/2015/06/Angela-Regina-Heinzen-Amin-Helou1.pdf. Acesso em: 29 set. 2016.

JUÁREZ, Hernándesz José Luis; CERVANTES, Baltazar Pérez. La gestión del conocimiento como estratégia para la mejora continua em la Administración Publica Municipal. La experiencia del H Ayuntamiento de Novolato. Internacional Jounal of Good Conscience, v. 7, n. 3, p. 1-14. nov./2012. Disponível em: http://www.spentamexico.org/v7-n3/7(3)1-14.pdf. Acesso em: 29 set. 2016.

KRAEMER, Rodrigo; FREIRE, Patrícia de Sá; SOUZA, João Artur; DANDOLINI, Gertrudes A. Maturidade de gestão do conhecimento: uma revisão sistemática da literatura para apoiar o desenvolvimento de novos modelos de avaliação. Perspectivas em Gestão \& Conhecimento, João Pessoa, v. 7, Número Especial, p. 66-79, mar. 2017. Disponível em: http://periodicos.ufpb.br/index.php/pgc/article/view/32946/17302. Acesso em: 1 maio 2017.

MARTÍNEZ, José Ángel; LARA-NAVARRA, Pabl; BELTRÁN, Pilar. La influencia de la sociedade del conocimiento em la modernización de la Administración Pública. Revista sobre la sociedade de conocimiento. UOC papers, n. 3, 2006. Disponível em: http://www.uoc.edu/uocpapers/3/dt/esp/martinez lara beltran.pdf. Acesso em: 29 set. 2016.

MENEZES, Katia Costa de; JOHANN, Juliana; VALENTIM, Patrícia Passeri; SCOTT, Patrícia. Gestão do Conhecimento nas Organizações: Uma aprendizagem em Rede Colaborativa. Perspectivas em Gestão \& Conhecimento, João Pessoa, v. 7, Número Especial, p. 145-159, mar. 2017. Disponível em: http://periodicos.ufpb.br/ojs2/index.php/pgc. Acesso em: 1 maio 2017.

OLIVEIRA, Míriam; PEDRON, Cristiane; ROMÃO, Mário; BECKER, Grace. Proposta de um modelo de maturidade para gestão do conhecimento: KM3. Revista Portuguesa e Brasileira de Gestão, out./dez., 2011.

PARDO, Sebastián; CORONEL, Juan Enrique; BERTONE, Rodolfo; THOMAS, Pablo. Gestión del Conocimiento: um enfoque aplicado em la Administración Pública. Argentina: Intituto de Investigación em Informática (LIDI) - Faculdade de Informática. Universidade Nacional de La Plata. 2013. Disponível em: http://www.lidi.info.unlp.edu.ar/wp/wpcontent/uploads/2013/09/CACIC2013PardoCoronelBertoneThomas.pdf. Acesso em: 29 set. 2016. 
RIBEIRO, Jurema Suely de Araújo Nery; SOARES, Marco Antônio Calijorne; JURZA, Paulo Henrique; ZIVIANI, Fabricio; NEVES, Jorge Tadeu de Ramos. Gestão do Conhecimento e Desenvolvimento Organizacional: Integração Dinâmica entre Competências e Recursos. Perspectivas em Gestão \& Conhecimento, João Pessoa, v. 7, número especial, p. 4-17, mar. 2017. Disponível em: http://periodicos.ufpb.br/index.php/pgc/article/viewFile/32936/17289. Acesso em: 1 maio 2017.

SANTOS, Vanessa dos; BASTOS, Rogério Cid. Gestão do conhecimento e administração pública: uma revisão sistemática da literatura. Revista Contribuciones a las Ciencias Sociales, eneromarzo, 2018. Disponível em: http://www.eumed.net/rev/cccss/2018/01/gestaoconhecimento-administracao.html. Acesso em: 07 set. 2018.

SEVERINO, Antônio Joaquim. Metodologia do trabalho científico. 23. ed. São Paulo: Cortez, 2007. 